\title{
Tectonic shortening and coeval volcanism during the Quaternary, Northeast Japan arc
}

\author{
Koji Umeda ${ }^{1, *}$, Masao Ban ${ }^{2}$, Shintaro Hayashi ${ }^{3}$ and Tomohiro Kusano ${ }^{1}$ \\ ${ }^{1}$ Tono Geoscientific Research Unit, Geological Isolation Research and Development Directorate, \\ Japan Atomic Energy Agency, 959-31, Jorinji, Izumi, Toki, 509-5102, Japan. \\ ${ }^{2}$ Department of Earth and Environmental Sciences, Yamagata University, 1-4-12 Kojirakawa-machi, \\ Yamagata, 990-8560, Japan. \\ ${ }^{3}$ Department of Natural and Environmental Sciences, Akita University, 1-1, Gakuen-machi, Tegata, \\ Akita, 010-8502, Japan. \\ *Corresponding author. e-mail: umeda.koji@jaea.go.jp
}

\begin{abstract}
The Northeast Japan arc, a mature volcanic arc with a back-arc marginal basin (Japan Sea), is located on a convergent plate boundary along the subducting Pacific plate and the overriding North American plate. From a compilation and analysis of stratigraphy, radiometric age and data on erupted magma volumes, 176 eruptive episodes identified from 69 volcanoes so far, indicate that notable changes in eruption style, magma discharge rates and distribution of eruptive centres occurred around 1.0 Ma. Before ca. 1.0 Ma, large-volume felsic eruptions were dominant, forming large calderas in the frontal arc, a region of low crustal strain rate. After ca. 1.0 Ma to the present, the calc-alkaline andesite magma eruptions in the frontal and rear arcs, synchronous with crustal shortening characterized by reverse faulting, resulted in stratovolcano development along narrow uplifted zones. Although, it is widely assumed that magma cannot rise easily in a compressional setting, some of the magma stored within basal sills could be extruded where $\mathrm{N}-\mathrm{S}$-trending uplifted mountains bounded by reverse faults formed since about ca. 1.0 Ma.
\end{abstract}

\section{Introduction}

At highly active compressive margins, such as the Northeast (NE) Japan arc, current stresses are mostly compressive and the greatest principal stress is horizontal, indicating that the major faults are thrust faults (e.g., Nakata and Imaizumi 2002). In compressional settings, it is considered that magma cannot ascend easily, favouring magma upwelling along vertical fractures perpendicular to the regional least principal stress $\left(\sigma_{3}\right)$ (Hubbert and Willis 1957). It has been suggested by many authors that volcanism should be rare if not absent under horizontal compression (e.g., Hamilton 1995;
Watanabe et al. 1999). However, volcanism is especially common in areas of extensional tectonics, and notably so at divergent plate boundaries (e.g., Galland et al. 2007). Similarly, numerous Quaternary volcanoes are distributed in the NE Japan arc, where the Pacific plate is subducting along the Japan Trench under the North American plate at a rate close to the modelled convergence of nearly $9 \mathrm{~cm} / \mathrm{yr}$ (DeMets 1992). Indeed, the global positioning system measurements reveal large contraction $(>0.1 \mathrm{ppm} / \mathrm{yr})$ under compression in the arc (e.g., Sagiya et al. 2000). This region is probably one of the most extensively studied volcanic arcs in the world, particularly regarding the relationship

Keywords. Northeast Japan arc; volcanism; tectonic shortening; Quaternary. 
between volcanism and tectonics, and high-quality geological, geophysical and geochemical data are readily available (e.g., Hasegawa et al. 1978; Zhao et al. 1992; Sato 1994; Umeda et al. 1999; Yoshida 2001; Tamura et al. 2002; Kimura and Yoshida 2006; Acocella et al. 2008).

It has recently been recognized that Quaternary volcanoes are clustered in regions trending transverse to their host arc axis, and separated by subparallel, volcano-free gaps $30-75 \mathrm{~km}$ wide along the arc (figure 1) (Umeda et al. 1999; Tamura et al. 2002). The arrangement of the volcanic clusters, coupled with topographic profiles and negative Bouguer anomalies, could be controlled by magma

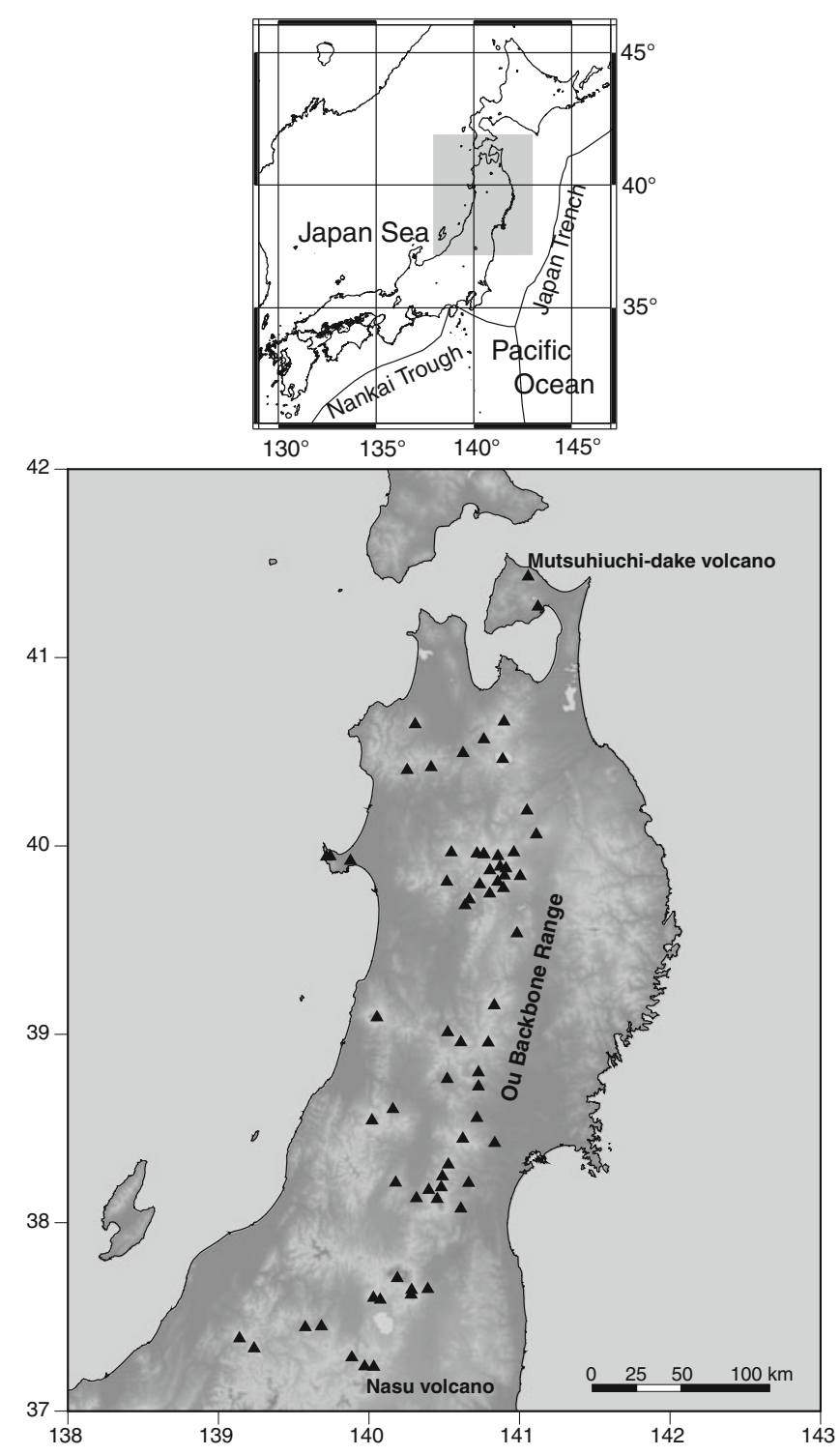

Figure 1. Location and shaded relief maps of NE Japan and distribution of Quaternary volcanoes (black triangles) and active faults (black lines) (Nakata and Imaizumi 2002). Solid triangles and open squares represent stratovolcanoes and large-scale caldera volcanoes, respectively. supplied from locally developed hot regions within the mantle wedge (Tamura et al. 2002). In the NE Japan arc, a change in the stress field at around the Pliocene to Quaternary boundary resulted in E-W compression (Sato 1994). In connection with the tectonic sequence, felsic volcanism associated with the large calderas terminated and andesite stratovolcanoes became the dominant form of volcanism during the Quaternary (Sato 1994). A compressive stress regime is not favourable to the development of felsic volcanism, requiring the formation of a large magma reservoir at shallow depth (Yoshida 2001). The predominance of stratovolcanoes can be reconciled with the crustal stress field in the present-day subduction system. In contrast, magma discharge rate is inferred to have increased in the NE Japan arc in the late Quaternary. A compilation of age data indicates that volcanoes ranging in age from 2.5 Ma to 1.5 Ma are relatively rare compared with those younger than 1.5 Ma (Kimura and Yoshida 2006). This insight appears to be contradictory to the idea that compressive stress in the Quaternary would prevent the rise of magma within the crust.

Recently, new stratigraphic and radiometric ages of Quaternary volcanoes in the NE Japan arc have been reported, and several synthesized databases were compiled by Umeda et al. (1999) and Committee for Catalogue of Quaternary Volcanoes in Japan (1999). Therefore, the special and temporal variations in eruption style, magma discharge rate and distribution of volcanoes during the Quaternary can be recognized with higher resolution than before. With the merging of this type of information, an understanding of the geometric and kinetic features of the structures controlling magma rise and emplacement in convergent settings can be developed. This understanding is also important to applied studies of natural hazards and economic resources. For this paper, we compiled the latest radiometric and stratigraphic data, and calculated the dense-rock equivalent (DRE) (Walker 1980) of volcanic products for each volcano. We describe time-magma volume behaviour that could provide insight into the tectono-magmatic relationships throughout the volcanic arc during the Quaternary. In addition, we examined the mechanism of magma transfer within the crust in a compressional setting.

\section{Geological background}

The Cenozoic volcanic and tectonic sequence in the NE Japan arc is directly associated with the separation of the present-day NE Japan arc from the Asian continental margin due to the subduction of the Pacific plate and rifting and opening 
Table 1. Ages and magma volume (DRE: dense rock equivalent) for each eruptive episode. Strat., stratigraphical method; TL, thermo-luminescence dating; $K-A r$, potassium argon dating; ${ }^{14} C$, radiocarbon dating; FT, fission track dating. (References for dating: (1) Umeda and Danhara 2008; (2) Arakawa et al. 2008; (3) Suzuki et al. 2005; (4) Committee for Catalog of Quaternary Volcanoes in Japan 1999; (5) Nozawa 2001; (6) Aoki and Arai 2000; (7) Ohba et al. 2003; (8) Kano et al. 2007; (9) Yashima 1990; (10) Fujinawa et al. 2001b; (11) Tsuchiya et al. 1997; (12) Mimura 2001; (13) Mimura and Kano 2000; (14) Umeda et al. 1999; (15) Fujinawa et al. 2001a; (16) Chiba and Kimura 2001; (17) Mimura 2002; (18) Yamamoto 2003, (19) Yamamoto and Komazawa 2004; (20) NEDO 1990).

\begin{tabular}{|c|c|c|c|c|c|c|c|}
\hline Volcano & Eruptive episode & Latitude & Longitude & Age (Ma) & Method & Ref. & $\begin{array}{c}\mathrm{DRE} \\
\left(\mathrm{km}^{3}\right)\end{array}$ \\
\hline Mutsuhiuchi-dake & Older Mutsuhiuchi-dake & 41.437 & 141.057 & $1.2-0.8$ & $\mathrm{~K}-\mathrm{Ar}$ & $(1)$ & 5.9 \\
\hline Mutsuhiuchi-dake & Younger Mutsuhiuchi-dake & 41.437 & 141.057 & $0.8-0.5$ & $\mathrm{~K}-\mathrm{Ar}, \mathrm{FT}$ & $(1)$ & 3.6 \\
\hline Osorezan & Kamabuse-yama & 41.277 & 141.123 & $0.80-0.76$ & $\mathrm{~K}-\mathrm{Ar}, \mathrm{FT}$ & $(2)$ & 5.6 \\
\hline Osorezan & Byobuyama-Asahinadake & 41.277 & 141.123 & $0.7-0.5$ & $\mathrm{~K}-\mathrm{Ar}, \mathrm{FT}$ & $(2)$ & 3.2 \\
\hline Osorezan & Pre-caldera pyroclastic flow & 41.277 & 141.123 & $0.48-0.27$ & $\mathrm{~K}-\mathrm{Ar}, \mathrm{FT}$ & $(2)$ & 6.4 \\
\hline Osorezan & Post-caldera pyroclastic flow & 41.277 & 141.123 & $0.27-0.20$ & $\mathrm{~K}-\mathrm{Ar}, \mathrm{FT}$ & $(2)$ & 1.3 \\
\hline Osorezan & Tsurugiyama & 41.277 & 141.123 & $0.2-0.08$ & $\mathrm{~K}-\mathrm{Ar}, \mathrm{FT}$ & $(2)$ & 0.1 \\
\hline Hakkoda & Hakkoda P.F. 1st. & 40.667 & 140.897 & 0.76 & Strat. & $(3)$ & 17.8 \\
\hline Hakkoda & South-Hakkoda & 40.600 & 140.850 & $0.65-0.4$ & $\mathrm{~K}-\mathrm{Ar}$ & $(4)$ & 52.4 \\
\hline Hakkoda & Hakkoda P.F. 2nd. & 40.667 & 140.897 & 0.4 & $\mathrm{~K}-\mathrm{Ar}$ & $(4)$ & 17.3 \\
\hline Hakkoda & North-Hakkoda & 40.650 & 140.883 & $0.16-0$ & $\mathrm{~K}-\mathrm{Ar}$ & $(4)$ & 30.4 \\
\hline Iwaki & Iwaki & 40.653 & 140.307 & $0.330-0.000$ & $\mathrm{~K}-\mathrm{Ar}$ & $(4)$ & 37.2 \\
\hline Okiura & AoniF.Aonigawa P.F. & 40.573 & 140.763 & ca. 1.7 & $\mathrm{~K}-\mathrm{Ar}$ & $(5)$ & 17.6 \\
\hline Okiura & AoniF.Other P.F. & 40.573 & 140.763 & $1.7-0.9$ & $\mathrm{~K}-\mathrm{Ar}$ & $(5)$ & 3.7 \\
\hline Okiura & Okogawasawalava & 40.579 & 140.759 & $0.9-0.65$ & Strat. & $(5)$ & 0.9 \\
\hline Okiura & Okiuradacite & 40.557 & 140.755 & $0.9-0.7$ & $\mathrm{~K}-\mathrm{Ar}$ & $(5)$ & 2.1 \\
\hline Ikarigaseki & Nijikai Tuff & 40.500 & 140.625 & ca. 2.0 & $\mathrm{~K}-\mathrm{Ar}$ & $(4)$ & 20.2 \\
\hline Ikarigaseki & Ajarayama & 40.490 & 140.600 & $1.91-1.89$ & $\mathrm{~K}-\mathrm{Ar}$ & $(4)$ & 2.1 \\
\hline Towada & Herai-dake & 40.450 & 141.000 & & & & 5.1 \\
\hline Towada & Ohanabe-yama & 40.500 & 140.883 & $0.4-0.05$ & $\mathrm{~K}-\mathrm{Ar}$ & $(4)$ & 8.9 \\
\hline Towada & Hakka & 40.417 & 140.867 & & & & 1.4 \\
\hline Towada & Towada Okuse & 40.468 & 140.888 & 0.055 & ${ }^{14} \mathrm{C}$ & $(4)$ & 4.8 \\
\hline Towada & Towada Ofudo & 40.468 & 140.888 & 0.030 & Strat. & $(6)$ & 22.1 \\
\hline Towada & Towada Hachinohe & 40.468 & 140.888 & 0.015 & Strat. & $(6)$ & 26.9 \\
\hline Towada & Post-calderacones & 40.457 & 140.913 & $0.013-0$ & Strat. & $(4)$ & 14.4 \\
\hline Tashiro & Tashiro & 40.425 & 140.413 & $0.600-0.470$ & $\mathrm{~K}-\mathrm{Ar}$ & $(4)$ & 6.8 \\
\hline Tashiro & Hirataki P.F. & 40.420 & 140.413 & $0.020-0.020$ & Strat. & $(4)$ & 0.3 \\
\hline Taira-Komagatake & Taira-Komagatake & 40.410 & 140.254 & $0.200-0.170$ & $\mathrm{~K}-\mathrm{Ar}$ & $(4)$ & 2.3 \\
\hline Inaniwa & Inaniwa & 40.195 & 141.050 & $7.000-2.700$ & $\mathrm{~K}-\mathrm{Ar}$ & $(4)$ & 10.6 \\
\hline Nanashigure & Nanashigure & 40.068 & 141.112 & $1.06-0.72$ & $\mathrm{~K}-\mathrm{Ar}$ & $(4)$ & 55.5 \\
\hline Megata & Megata & 39.952 & 139.742 & $0.030-0.020$ & Strat. & $(4)$ & 0.0 \\
\hline Toga & Toga & 39.950 & 139.718 & ca. 0.42 & $\mathrm{FT} / \mathrm{K}-\mathrm{Ar}$ & $(4)$ & 1.2 \\
\hline Kampu & Kampu & 39.928 & 139.877 & $0.030-0.000$ & Strat. & $(4)$ & 0.5 \\
\hline Moriyoshi & Moriyoshi & 39.973 & 140.547 & $1.07-0.78$ & $\mathrm{~K}-\mathrm{Ar}$ & $(4)$ & 18.1 \\
\hline Bunamori & Bunamori & 39.967 & 140.717 & 1.2 & $\mathrm{~K}-\mathrm{Ar}$ & $(4)$ & 0.1 \\
\hline Akita-Yakeyama & Akita-Yakeyama & 39.963 & 140.763 & $0.5-0$ & $\mathrm{~K}-\mathrm{Ar}$ & $(4)$ & 9.9 \\
\hline Nishimori/Maemori & Nishimori/Maemori & 39.973 & 140.962 & $0.5-0.3$ & $\mathrm{~K}-\mathrm{Ar}$ & $(4)$ & 2.6 \\
\hline Hachimantai/Chausu & Hachimantai & 39.953 & 140.857 & $1.0-0.7$ & $\mathrm{~K}-\mathrm{Ar}$ & $(4)$ & 5.5 \\
\hline Hachimantai/Chausu & Chausu-dake & 39.948 & 140.902 & $0.85-0.75$ & $\mathrm{~K}-\mathrm{Ar}$ & $(4)$ & 13.7 \\
\hline Hachimantai/Chausu & Fukenoyu & 39.953 & 140.857 & ca. 0.7 & Strat. & $(4)$ & 0.2 \\
\hline Hachimantai/Chausu & Gentamri & 39.956 & 140.878 & & $(4)$ & 0.2 & \\
\hline Yasemori/Magarisaki-yama & Magarisaki-yama & 39.878 & 140.803 & $1.9-1.52$ & $\mathrm{~K}-\mathrm{Ar}$ & $(4)$ & 0.3 \\
\hline Yasemori/Magarisaki-yama & Yasemori & 39.883 & 140.828 & 1.8 & $\mathrm{~K}-\mathrm{Ar}$ & $(4)$ & 0.9 \\
\hline Kensomori/Morobidake & Kensomori & 39.897 & 140.871 & ca. 0.8 & Strat. & $(4)$ & 0.8 \\
\hline Kensomori/Morobidake & Morobi-dake & 39.919 & 140.862 & $1.0-0.8$ & Strat. & $(4)$ & 2.5 \\
\hline Kensomori/Morobidake & 1470 m Mt. lava & 39.909 & 140.872 & 0.72 & $\mathrm{~K}-\mathrm{Ar}$ & $(7)$ & 0.1 \\
\hline Kensomori/Morobidake & Mokko-dake & 39.953 & 140.857 & ca. 1.0 & Strat. & $(4)$ & 0.5 \\
\hline Tamagawa Welded Tuff & Tamagawa Welded Tuffs R4 & 39.963 & 140.763 & ca. 2.0 & $\mathrm{~K}-\mathrm{Ar}$ & $(4)$ & 83.2 \\
\hline Tamagawa Welded Tuff & Tamagawa Welded Tuffs D & 39.963 & 140.763 & ca. 1.0 & $\mathrm{~K}-\mathrm{Ar}$ & $(4)$ & 32.0 \\
\hline
\end{tabular}


Table 1. (Continued)

\begin{tabular}{|c|c|c|c|c|c|c|c|}
\hline Volcano & Eruptive episode & Latitude & Longitude & Age (Ma) & Method & Ref. & $\begin{array}{l}\mathrm{DRE} \\
\left(\mathrm{km}^{3}\right)\end{array}$ \\
\hline Nakakura/Shimokura & Obuka-dake & 39.878 & 140.883 & $0.8-0.7$ & $\mathrm{~K}-\mathrm{Ar}$ & $(4)$ & 2.9 \\
\hline Nakakura/Shimokura & Shimokura-yama & 39.889 & 140.933 & $0.85-0.58$ & $\mathrm{~K}-\mathrm{Ar}$ & $(7)$ & 0.4 \\
\hline Nakakura/Shimokura & Nakakura-yama & 39.888 & 140.910 & $0.85-0.58$ & $\mathrm{~K}-\mathrm{Ar}$ & $(7)$ & 0.4 \\
\hline Matsukawa & Matsukawa andesite & 39.850 & 140.900 & $2.6-1.29$ & $\mathrm{~K}-\mathrm{Ar}$ & (4) & 11.6 \\
\hline Iwate/Amihari & Iwate & 39.847 & 141.004 & $0.2-0$ & $\mathrm{~K}-\mathrm{Ar}$ & $(4)$ & 25.1 \\
\hline Iwate/Amihari & Amihari & 39.842 & 140.958 & $0.3-0.1$ & $\mathrm{~K}-\mathrm{Ar}$ & $(4)$ & 10.6 \\
\hline Iwate/Amihari & Omatsukura-yama & 39.841 & 140.919 & $0.7-0.6$ & $\mathrm{~K}-\mathrm{Ar}$ & $(4)$ & 3.3 \\
\hline Iwate/Amihari & Kurikigahara & 39.849 & 140.882 & & & & 0.2 \\
\hline Iwate/Amihari & Mitsuishi-yama & 39.848 & 140.900 & 0.46 & $\mathrm{~K}-\mathrm{Ar}$ & $(4)$ & 0.6 \\
\hline Shizukuishi/Takakura & Marumori & 39.775 & 140.877 & $0.4-0.3$ & $\mathrm{~K}-\mathrm{Ar}$ & (4) & 2.4 \\
\hline Shizukuishi/Takakura & Shizukuishi-Takakura-yama & 39.783 & 140.893 & $0.5-0.4$ & Strat. & (4) & 5.2 \\
\hline Shizukuishi/Takakura & Older Kotakakura-yama & 39.800 & 140.900 & 1.4 & $\mathrm{~K}-\mathrm{Ar}$ & (4) & 2.7 \\
\hline Shizukuishi/Takakura & North Mikado-yama & 39.800 & 140.875 & & & & 0.3 \\
\hline Shizukuishi/Takakura & Kotakakura-yama & 39.797 & 140.907 & $0.6-0.5$ & $\mathrm{~K}-\mathrm{Ar}$ & $(4)$ & 1.8 \\
\hline Shizukuishi/Takakura & Mikado-yama & 39.788 & 140.870 & ca. 0.3 & Strat. & $(4)$ & 0.2 \\
\hline Shizukuishi/Takakura & Tairagakura-yama & 39.808 & 140.878 & ca. 0.3 & Strat. & $(4)$ & 0.1 \\
\hline Nyuto/Zarumori & Tashirotai & 39.812 & 140.827 & $0.3-0.2$ & $\mathrm{~K}-\mathrm{Ar}$ & $(4)$ & 0.6 \\
\hline Nyuto/Zarumori & Sasamori-yama & 39.770 & 140.820 & $0.23-0.1$ & $\mathrm{~K}-\mathrm{Ar}$ & (4) & 0.4 \\
\hline Nyuto/Zarumori & Yunomori-yama & 39.772 & 140.827 & ca. 0.3 & & $(4)$ & 0.5 \\
\hline Nyuto/Zarumori & Zarumori-yama & 39.788 & 140.850 & 0.56 & $\mathrm{~K}-\mathrm{Ar}$ & $(4)$ & 0.9 \\
\hline Nyuto/Zarumori & Nyutozan & 39.802 & 140.843 & $0.58-0.5$ & $\mathrm{~K}-\mathrm{Ar}$ & $(4)$ & 5.0 \\
\hline Nyuto/Zarumori & Nyuto-kita & 39.817 & 140.855 & ca. 0.4 & $\mathrm{~K}-\mathrm{Ar}$ & $(4)$ & 0.1 \\
\hline Akita-Komagatake & Akita-Komagatake & 39.754 & 140.802 & $0.1-0$ & $\mathrm{~K}-\mathrm{Ar}$ & (4) & 2.9 \\
\hline Kayo & Kayo & 39.803 & 140.735 & $2.2-1.17$ & $\mathrm{~K}-\mathrm{Ar}$ & $(4)$ & 5.9 \\
\hline Kayo & KoJiromori & 39.828 & 140.787 & 0.94 & $\mathrm{~K}-\mathrm{Ar}$ & (4) & 0.3 \\
\hline Kayo & Akita-Ojiromori & 39.839 & 140.788 & 1.7 & Strat. & (4) & 0.3 \\
\hline Daibutsu & Daibutsu & 39.817 & 140.517 & $2.34-2.16$ & $\mathrm{~K}-\mathrm{Ar}$ & $(4)$ & 2.4 \\
\hline Tazawa & Tazawa & 39.723 & 140.667 & $1.80-1.40$ & $\mathrm{FT}$ & $(8)$ & \\
\hline Innai/Takahachi & Takahachi-yama & 39.755 & 140.655 & 1.7 & $\mathrm{~K}-\mathrm{Ar}$ & $(4)$ & 0.0 \\
\hline Innai/Takahachi & Innai & 39.692 & 140.638 & $2.0-1.6$ & $\mathrm{~K}-\mathrm{Ar}$ & $(4)$ & 0.5 \\
\hline Kuzumaru & Aonokimori andesites & 39.543 & 140.983 & 2.06 & $\mathrm{~K}-\mathrm{Ar}$ & $(9)$ & 0.3 \\
\hline Yakeishi & Yakeishidake & 39.161 & 140.832 & $0.7-0.6$ & $\mathrm{~K}-\mathrm{Ar}$ & $(4)$ & 9.5 \\
\hline Yakeishi & Komagatake & 39.193 & 140.924 & ca. 1.0 & $\mathrm{~K}-\mathrm{Ar}$ & $(4)$ & 7.6 \\
\hline Yakeishi & Kyozukayama & 39.178 & 140.892 & $0.6-0.4$ & $\mathrm{~K}-\mathrm{Ar}$ & (4) & 5.7 \\
\hline Yakeishi & Usagimoriyama & 39.239 & 140.924 & $0.07-0.04$ & $\mathrm{~K}-\mathrm{Ar}$ & $(4)$ & 2.3 \\
\hline Chokai & Shinsan Lava flow & 39.097 & 140.053 & $0.02-0$ & Strat. & $(4)$ & 0.6 \\
\hline Chokai & Higashi Chokai & 39.097 & 140.053 & $0.02-0.02$ & $\mathrm{~K}-\mathrm{Ar}$ & $(4)$ & 3.3 \\
\hline Chokai & Nishi Chokai & 39.097 & 140.020 & $0.09-0.02$ & Stra. & $(4)$ & 0.6 \\
\hline Chokai & Nishi Chokai II & 39.097 & 140.020 & $0.13-0.01$ & $\mathrm{~K}-\mathrm{Ar}$ & $(4)$ & 16.0 \\
\hline Chokai & Old Chokai & 39.103 & 140.030 & $0.55-0.16$ & $\mathrm{~K}-\mathrm{Ar}$ & $(4)$ & 50.9 \\
\hline Chokai & Uguisugawa Basalt & 39.103 & 140.030 & $0.6-0.55$ & $\mathrm{~K}-\mathrm{Ar}$ & $(4)$ & 0.8 \\
\hline Chokai & Tengumari volcanics & 39.103 & 140.031 & $0.6-0.55$ & $\mathrm{~K}-\mathrm{Ar}$ & $(4)$ & 8.4 \\
\hline Kobinai & Kobinai & 39.018 & 140.523 & $1.0-0.57$ & $\mathrm{~K}-\mathrm{Ar}, \mathrm{FT}$ & $(4)$ & 2.3 \\
\hline Takamatsu/Kabutoyama & Kabutoyama Welded Tuffs & 39.025 & 140.618 & 1.16 & TL & (4) & 3.2 \\
\hline Takamatsu/Kabutoyama & Kiji-yama Welded Tuffs & 39.025 & 140.618 & 0.30 & $\mathrm{~K}-\mathrm{Ar}$ & $(4)$ & 5.1 \\
\hline Takamatsu & Takamatsu & 38.965 & 140.610 & $0.3-0.27$ & $\mathrm{~K}-\mathrm{Ar}$ & $(4)$ & 3.8 \\
\hline Takamatsu & Futsutsuki-dake & 38.961 & 140.661 & ca. 0.3 & & $(4)$ & 0.8 \\
\hline Kurikoma & Tsurugi-dake & 38.963 & 140.792 & $0.1-0$ & $\mathrm{~K}-\mathrm{Ar}$ & $(10)$ & 0.2 \\
\hline Kurikoma & Magusa-dake & 38.968 & 140.751 & $0.32-0.1$ & $\mathrm{~K}-\mathrm{Ar}$ & $(10)$ & 1.5 \\
\hline Kurikoma & Kurikoma & 38.963 & 140.792 & $0.4-0.1$ & $\mathrm{~K}-\mathrm{Ar}$ & $(10)$ & 0.9 \\
\hline Kurikoma & South volcanoes & 38.852 & 140.875 & ca. 0.5 & $\mathrm{~K}-\mathrm{Ar}$ & $(10)$ & 0.3 \\
\hline Kurikoma & Older Higashi Kurikoma & 38.934 & 140.779 & ca. 0.5 & $\mathrm{~K}-\mathrm{Ar}$ & $(10)$ & 2.2 \\
\hline Kurikoma & Younger Higashi Kurikoma & 38.934 & 140.779 & $0.4-0.1$ & $\mathrm{~K}-\mathrm{Ar}$ & $(10)$ & 0.7 \\
\hline
\end{tabular}


Table 1. (Continued)

\begin{tabular}{|c|c|c|c|c|c|c|c|}
\hline Volcano & Eruptive episode & Latitude & Longitude & Age (Ma) & Method & Ref. & $\begin{array}{l}\mathrm{DRE} \\
\left(\mathrm{km}^{3}\right)\end{array}$ \\
\hline Mukaimachi & Mukaimachi & 38.770 & 140.520 & ca. 0.8 & $\mathrm{~K}-\mathrm{Ar}$ & $(4)$ & 12.0 \\
\hline Onikobe & Shimoyamasato Tuff & 38.830 & 140.695 & 0.21 & FT & $(4)$ & 1.0 \\
\hline Onikobe & Onikobe Central cones & 38.805 & 140.727 & ca. 0.2 & TL & (4) & 1.1 \\
\hline Onikobe & Ikezuki Tuff & 38.830 & 140.695 & $0.3-0.2$ & $\mathrm{FT}$ & $(4)$ & 17.3 \\
\hline Naruko & Naruko Central cones & 38.730 & 140.727 & 0.045 & ${ }^{14} \mathrm{C}$ & (4) & 0.1 \\
\hline Naruko & Yanagizawa Tuff & 38.730 & 140.727 & 0.045 & $\mathrm{FT}$ & $(4)$ & 4.8 \\
\hline Naruko & Nizaka Tuff & 38.730 & 140.727 & 0.073 & $\mathrm{FT}$ & $(4)$ & 4.8 \\
\hline Hijiori & Hijiori Pyroclastic flow & 38.610 & 140.159 & ca. 0.01 & Strat. & $(4)$ & 0.5 \\
\hline Hijiori & Komatsubuchi lava dome & 38.613 & 140.171 & ca. 0.01 & Strat. & (4) & 0.0 \\
\hline Gassan & Ubagatake & 38.533 & 140.005 & $0.400-0.300$ & $\mathrm{~K}-\mathrm{Ar}$ & $(4)$ & 2.7 \\
\hline Gassan & Yudonosanlavas/pyroclastics & 38.534 & 139.988 & $0.800-0.700$ & $\mathrm{~K}-\mathrm{Ar}$ & $(4)$ & 5.7 \\
\hline Gassan & Gassan & 38.550 & 140.020 & $0.500-0.400$ & $\mathrm{~K}-\mathrm{Ar}$ & $(4)$ & 13.7 \\
\hline Funagata & Izumigatake & 38.408 & 140.712 & $1.45-1.14$ & $\mathrm{~K}-\mathrm{Ar}$ & $(4)$ & 2.3 \\
\hline Funagata & Funagatayama & 38.453 & 140.623 & $0.85-0.56$ & $\mathrm{~K}-\mathrm{Ar}$ & $(4)$ & 19.0 \\
\hline Yakuraisan & Yakuraisan & 38.563 & 140.717 & $1.65-1.04$ & $\mathrm{~K}-\mathrm{Ar}$ & $(11)$ & 0.2 \\
\hline Nanatsumori & Nanatsumori lava & 38.430 & 140.835 & $2.3-2.0$ & $\mathrm{~K}-\mathrm{Ar}$ & $(12)$ & 0.5 \\
\hline Nanatsumori & Miyatoko Tuffs & 38.428 & 140.793 & ca. 2.5 & Strat. & $(4)$ & 6.1 \\
\hline Nanatsumori & Akakuzure-yama lava & 38.433 & 140.768 & $1.6-1.5$ & Strat. & $(4)$ & 1.5 \\
\hline Nanatsumori & Kamikadajin lava & 38.447 & 140.772 & $1.6-1.5$ & $\mathrm{~K}-\mathrm{Ar}$ & $(4)$ & 0.8 \\
\hline Shirataka & Shirataka & 38.220 & 140.177 & $1.0-0.8$ & $\mathrm{~K}-\mathrm{Ar}$ & (13) & 3.8 \\
\hline Adachi & Adachi & 38.218 & 140.662 & 0.08 & $\mathrm{FT}$ & $(4)$ & 0.9 \\
\hline Gantosan & Gantosan & 38.195 & 140.480 & $0.4-0.3$ & $\mathrm{~K}-\mathrm{Ar}$ & $(4)$ & 4.6 \\
\hline Kamuro-dake & Kamuro-dake & 38.253 & 140.488 & 1.67 & $\mathrm{~K}-\mathrm{Ar}$ & $(12)$ & 5.7 \\
\hline Daito-dake & Daito-dake & 38.316 & 140.527 & ca. 1.0 & Strat. & (4) & 5.7 \\
\hline Sankichi-Hayama & Sankichi-Hayama & 38.137 & 140.315 & $2.400-2.300$ & $\mathrm{~K}-\mathrm{Ar}$ & $(4)$ & 2.2 \\
\hline Ryuzan & Ryuzan & 38.181 & 140.397 & $1.1-0.9$ & $\mathrm{~K}-\mathrm{Ar}$ & (4) & 4.6 \\
\hline Zao & Central Zao 1st. & 38.133 & 140.453 & $1.46-0.79$ & $\mathrm{~K}-\mathrm{Ar}$ & $(14)$ & 0.8 \\
\hline Zao & Central Zao 2nd. & 38.133 & 140.453 & $0.32-0.12$ & $\mathrm{~K}-\mathrm{Ar}$ & $(14)$ & 15.2 \\
\hline Zao & Central Zao 3rd. & 38.133 & 140.453 & $0.03-0$ & $\mathrm{~K}-\mathrm{Ar}$ & $(14)$ & 0.0 \\
\hline $\mathrm{Zao}$ & Sugigamine & 38.103 & 140.462 & 1.0 & $\mathrm{~K}-\mathrm{Ar}$ & (14) & 9.9 \\
\hline $\mathrm{Zao}$ & Fubosan/byobudake & 38.093 & 140.478 & $0.31-0.17$ & $\mathrm{~K}-\mathrm{Ar}$ & (14) & 15.2 \\
\hline Aoso-yama & Gairinzan & 38.082 & 140.610 & $0.7-0.4$ & $\mathrm{~K}-\mathrm{Ar}$ & $(12)$ & 6.1 \\
\hline Aoso-yama & Central Cone & 38.082 & 140.610 & $0.40-0.3$ & $\mathrm{~K}-\mathrm{Ar}$ & $(4)$ & 3.0 \\
\hline Azuma & Azuma Kiteilava & 37.733 & 140.247 & $1.3-1.0$ & $\mathrm{~K}-\mathrm{Ar}$ & (4) & 24.7 \\
\hline Azuma & Higashi Azumasan & 37.710 & 140.233 & $0.7-0$ & $\mathrm{~K}-\mathrm{Ar}$ & $(4)$ & 22.8 \\
\hline Azuma & Nishi Azumasan & 37.730 & 140.150 & $0.6-0.4$ & $\mathrm{~K}-\mathrm{Ar}$ & $(4)$ & 7.2 \\
\hline Azuma & Naka Azumasan & 37.713 & 140.188 & $0.4-0.3$ & $\mathrm{~K}-\mathrm{Ar}$ & $(4)$ & 4.6 \\
\hline Nishikarasugawaandesite & Nishikarasugawa andesite & 37.650 & 140.283 & ca. 1.5 & $\mathrm{~K}-\mathrm{Ar}$ & $(4)$ & 1.9 \\
\hline Adatara & Adatara Stage 1 & 37.625 & 140.280 & $0.55-0.44$ & $\mathrm{~K}-\mathrm{Ar}$ & $(15)$ & 0.3 \\
\hline Adatara & Adatara Stage 2 & 37.625 & 140.280 & ca. 0.35 & $\mathrm{~K}-\mathrm{Ar}$ & $(15)$ & 0.4 \\
\hline Adatara & Adatara Stage 3a & 37.625 & 140.280 & ca. 0.20 & $\mathrm{~K}-\mathrm{Ar}$ & $(15)$ & 2.0 \\
\hline Adatara & Adatara Stage 3b & 37.625 & 140.280 & $0.12-0.002$ & $\mathrm{~K}-\mathrm{Ar}$ & $(15)$ & 0.3 \\
\hline Sasamori-yama & Sasamari-yama andesite & 37.655 & 140.391 & $2.5-2$ & $\mathrm{~K}-\mathrm{Ar}$ & $(4)$ & 0.4 \\
\hline Bandai & Pre-Bandai & 37.598 & 140.075 & ca. 0.7 & $\mathrm{~K}-\mathrm{Ar}$ & $(4)$ & 0.1 \\
\hline Bandai & Bandai & 37.598 & 140.075 & $0.3-0$ & Strat. & $(16)$ & 14.0 \\
\hline Nekoma & Old Nekoma & 37.608 & 140.030 & $1.0-0.7$ & $\mathrm{~K}-\mathrm{Ar}$ & $(17)$ & 11.4 \\
\hline Nekoma & New Nekoma & 37.608 & 140.030 & $0.5-0.4$ & $\mathrm{~K}-\mathrm{Ar}$ & $(17)$ & 0.9 \\
\hline Numazawa & Shirifukitoge P. & 37.452 & 139.577 & 0.11 & $\mathrm{FT}$ & (18) & 0.7 \\
\hline Numazawa & Mukuresawa L. & 37.452 & 139.577 & 0.071 & TL & $(18)$ & 0.1 \\
\hline Numazawa & Mizunuma P. & 37.452 & 139.577 & 0.045 & ${ }^{14} \mathrm{C}$ & $(18)$ & 1.0 \\
\hline Numazawa & Sozan L. & 37.452 & 139.577 & 0.043 & $\mathrm{FT}$ & $(18)$ & 0.3 \\
\hline Numazawa & Numagozen P. & 37.452 & 139.577 & 0.0198 & ${ }^{14} \mathrm{C}$ & (18) & 0.0 \\
\hline Numazawa & Maeyama L. & 37.452 & 139.577 & 0.02 & ${ }^{14} \mathrm{C}$ & (18) & 0.3 \\
\hline
\end{tabular}


Table 1. (Continued)

\begin{tabular}{|c|c|c|c|c|c|c|c|}
\hline Volcano & Eruptive episode & Latitude & Longitude & Age (Ma) & Method & Ref. & $\begin{array}{l}\mathrm{DRE} \\
\left(\mathrm{km}^{3}\right)\end{array}$ \\
\hline Numazawa & Numazawako P. & 37.452 & 139.577 & 0.005 & ${ }^{14} \mathrm{C}$ & $(18)$ & 2.0 \\
\hline Sunagohara & Sunagohara & 37.457 & 139.684 & $0.29-0.22$ & FT & (19) & 2.7 \\
\hline Sumon & Sumon & 37.393 & 139.140 & $2.40-1.75$ & $\mathrm{~K}-\mathrm{Ar}$ & $(4)$ & 22.0 \\
\hline Asakusa & Asakusa & 37.340 & 139.237 & $1.64-1.54$ & $\mathrm{~K}-\mathrm{Ar}$ & $(4)$ & 4.6 \\
\hline Kasshi/Oshiromori & Kasshi & 37.184 & 139.973 & & & & 0.1 \\
\hline Kasshi/Oshiromori & Oshiromori & 37.199 & 139.970 & & & & 0.7 \\
\hline Kasshi/Oshiromori & Matami-yama & 37.292 & 139.886 & 0.94 & $\mathrm{~K}-\mathrm{Ar}$ & $(20)$ & 0.3 \\
\hline Kasshi/Oshiromori & Naka-yama & 37.282 & 139.899 & & & & 0.0 \\
\hline Shirakawa & Kumado P.F. & 37.242 & 140.032 & 1.31 & $\mathrm{~K}-\mathrm{Ar}$ & $(4)$ & 19.2 \\
\hline Shirakawa & Tokaichi A.F. tuffs & 37.242 & 140.032 & $1.31-1.24$ & Strat. & $(4)$ & 12.0 \\
\hline Shirakawa & Ashino P.F. & 37.242 & 140.032 & 1.2 & FT & (4) & 19.2 \\
\hline Shirakawa & Nn3 P.F. & 37.242 & 140.032 & $1.20-1.17$ & Strat. & $(4)$ & 0.0 \\
\hline Shirakawa & Kinshoji A.F. tuffs & 37.242 & 140.032 & $1.20-1.18$ & Strat. & (4) & 9.0 \\
\hline Shirakawa & Nishigo P.F. & 37.252 & 139.869 & 1.11 & $\mathrm{FT}$ & $(4)$ & 28.8 \\
\hline Shirakawa & Tenei P.F. & 37.242 & 140.032 & 1.06 & Strat. & (4) & 7.7 \\
\hline Nasu & Futamata-yama & 37.244 & 139.971 & 0.14 & $\mathrm{~K}-\mathrm{Ar}$ & $(4)$ & 3.2 \\
\hline Nasu & Kasshiasahi-dake & 37.177 & 139.963 & $0.6-0.4$ & $\mathrm{~K}-\mathrm{Ar}$ & $(4)$ & 12.3 \\
\hline Nasu & Sanbonyari-dake & 37.147 & 139.965 & $0.4-0.25$ & $\mathrm{~K}-\mathrm{Ar}$ & $(4)$ & 5.5 \\
\hline Nasu & Minamigassan & 37.123 & 139.967 & $0.2-0.05$ & $\mathrm{~K}-\mathrm{Ar}$ & $(4)$ & 8.7 \\
\hline Nasu & Asahi-dake & 37.134 & 139.971 & $0.2-0.05$ & $\mathrm{~K}-\mathrm{Ar}$ & $(4)$ & 4.6 \\
\hline Nasu & Chausu-dake & 37.122 & 139.966 & $0.04-0$ & $\mathrm{~K}-\mathrm{Ar}$ & (4) & 0.3 \\
\hline
\end{tabular}

of the Japan Sea. The main rifting started at $\sim 23$ Ma (Taira 2001). Between 21 and 18 Ma, rifting was accompanied by significant counterclockwise rotation of the NE Japan arc (Jolivet et al. 1994). Owing to the cessation of the opening of the Japan Sea, the extensional stress field changed at about $13 \mathrm{Ma}$. From the Middle Miocene to the Pliocene, the tectonics is characterized by very weak crustal deformation under the moderate regional stress field related to the convergence of the Pacific plate (Sato 1994). The maximum horizontal stress oriented in the NE or ENE direction was manifested during this period. This is one of the reasons why the SW migration of the Kuril sliver due to the oblique convergence along the Kuril arc results from a NE or ENE trending maximum compression (e.g., Otsuki 1990). In relation to the stress change from extensional to a slightly compressive stress, a major variation in the volcanism in the NE Japan arc changed from rift-type into island-arc type in the last $13 \mathrm{Ma}$ (Sato 1994; Yoshida 2001), resulting in changes in the location and orientation of the volcanic front (Ohguchi et al. 1989). Submarine basaltic to rhyolitic volcanism was predominant from 13 to 8 Ma. Many Valles-type caldera (volcanic crater formed by collapse during an ignimbrite eruption) formed on the central mountains range along the arc since ca. $8 \mathrm{Ma}$. The dome-like structures around the calderas indicate that felsic magmatism in the shallow crust contributed to the gentle uplift of the central mountains range (Yoshida 2001).

Tectonic shortening of the crust due to E-W compression became apparent at about the Pliocene to Quaternary boundary, which may be associated with the increased motion of the Pacific Plate between 5 and $2 \mathrm{Ma}$ (Cox and Engebretson 1985; Pollitz 1986). A compressional stress field during the Quaternary is responsible for the development of two narrow uplift zones oriented in a $\mathrm{N}-\mathrm{S}$ direction, in the NE Japan arc: the Ou Backbone Range (fore-arc) and the Dewa Hills (reararc). They appear to be an active pop-up structure bounded by opposite-facing reverse faults accommodating $<5 \mathrm{~mm} / \mathrm{yr}$ of $\mathrm{E}-\mathrm{W}$ shortening across the range (Hasegawa et al. 2005). Based on the subsurface geology and deformation of river terraces, the initiation time of reverse faulting was estimated at several sites in the NE Japan. These results suggest that reverse faulting started on the rear-arc side between 3.4 and $2.4 \mathrm{Ma}$ (Awata and Kakimi 1985), and in the fore-arc side between 0.9 and 0.5 Ma (Otsuki et al. 1977), corresponding to the onset time of uplift of the Dewa Hills and the $\mathrm{Ou}$ Backbone Range. The compressional regime reactivated originally normal faults related to the extensional back-arc rifting as reverse faults and these accommodate much of the ongoing shortening across the arc. Andesite stratovolcanoes started to form between 2.5 and $1.5 \mathrm{Ma}$, but these are 
relatively rare compared with those younger $1.5 \mathrm{Ma}$ volcanoes (Kimura and Yoshida 2006). Nevertheless, it remains obscure as to when the calc-alkaline andesite volcanism, derived from the mixing between the felsic and mafic magmas (e.g., Sakuyama and Nesbitt 1986), has been established under Quaternary orthogonal convergence in the NE Japan arc.

\section{Estimation of timing and volume for each eruptive episode}

Although 34 Quaternary volcanoes have been recognized along the volcanic arc between Mutsuhiuchidake volcano and Nasu volcano in the NE Japan arc (Ono et al. 1981), they are predominantly polygenic, with new edifices forming at new locations and evolving into polygenic volcano centres. Before refining the sequence of volcanism during the Quaternary, we subdivided individual volcanoes identified by Ono et al. (1981) into as small a units as possible. The term 'eruptive episode' used herein refers to any unit that formed due to a series of eruptions from the same conduit or edifice, including monogenic volcanoes (volcano constructed during a single phase of eruptive activities) that formed in a relatively short time period and polygenetic volcanoes (resulting in from more than one formation process) that developed over several tens to hundreds of thousands of years. The active time span of eruptive episode with several radiometric ages can be estimated by their median age. Radiometric ages that are inconsistent with the stratigraphic data were discarded.

The magma volume discharged for each eruptive episode was calculated as follows: Assuming that a edifice is represented by a cone with a radius of basal circle $(R)$ and height between the base and apex $(H)$, volume $(V)$ is calculated by the following equation: $V=(1 / 3) H \pi R^{2}$. When it is represented by an elliptical cone, the radius of a basal circle $\left(R^{\prime}\right)$ with the same area as the basal ellipse is found, and then the $R^{\prime}$ is applied in the above equation (Umeda et al. 1999). The relief of the underlying basement can be considered. For example, when the basement of the conical edifice is uplifted in the central part to form a raised bottom, the excessive volume is subtracted from the conical volume. The volume of lava flows or pyroclastic flows is calculated by multiplying their basal area and average thickness. When it is difficult to estimate the average thickness, their distribution area is subdivided into smaller areas by taking into account basement relief, and the products of their area and average thickness in the respective subdivided areas are totalled. The DRE of erupted volumes was calculated by the product of volume and density of the respective volcanic products. Density values used are: stratovolcanoes, $1.9 \mathrm{~g} / \mathrm{cm}^{3}$; non-welded pyroclastic flow deposits, $1.2 \mathrm{~g} / \mathrm{cm}^{3}$; welded pyroclastic flow deposits, $1.6 \mathrm{~g} / \mathrm{cm}^{3}$; pyroclastic fall deposits, $1.5 \mathrm{~g} / \mathrm{cm}^{3}$ (Nakamura 1964; Smith 1979; Yoshida and Takahashi 1991).

Table 1 presents the locations, active time spans (range), and magma volumes of the individual eruptive episodes during the Quaternary, including the Gelasian Stage. Sixty-nine volcanoes composed of 176 eruptive episodes can be identified along the volcanic arc between Mutsuhiuchi-dake volcano and Nasu volcano (figure 1).

\section{Time-volume relationships for NE Japan volcanic arc since $2.0 \mathrm{Ma}$}

In order to elucidate temporal variations in the longterm magma discharge rate for all of the NE Japan

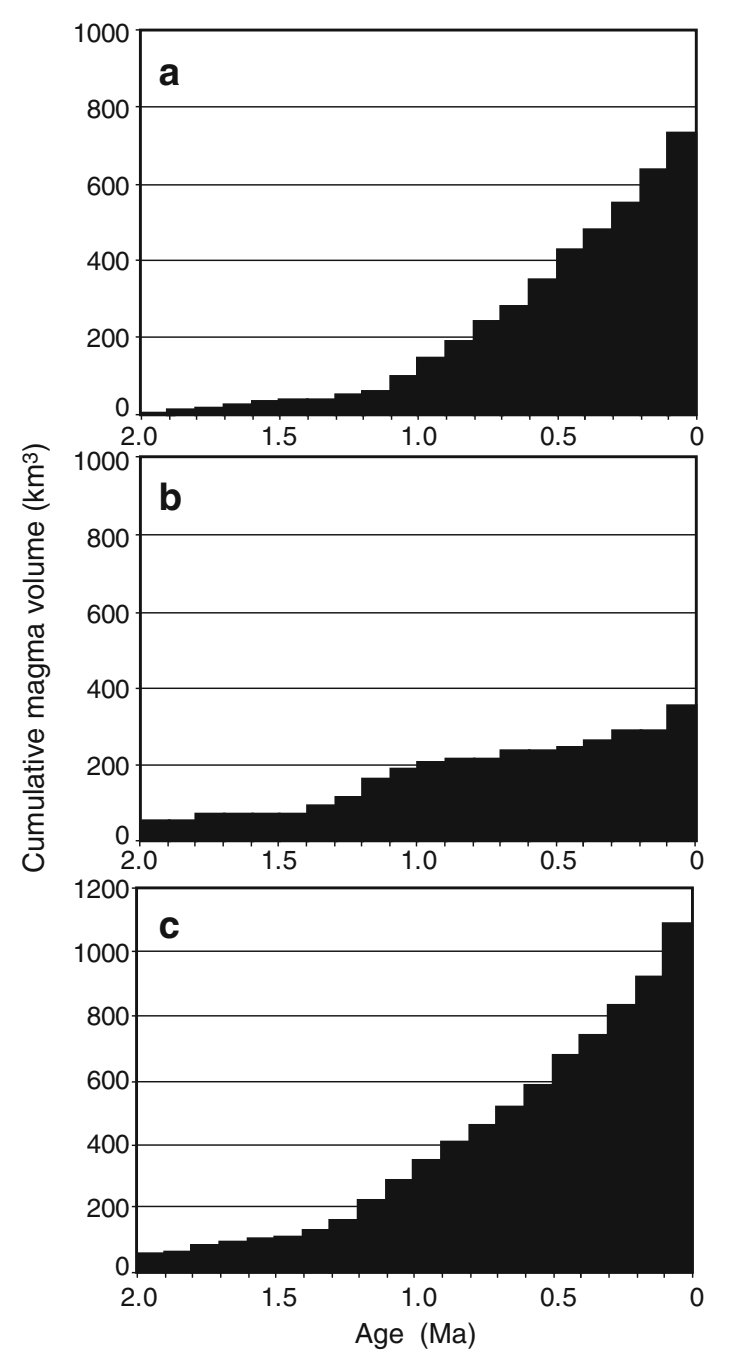

Figure 2. Time-volume relationships for the NE Japan volcanic arc since 2.0 Ma. (a) Volcanism associated with stratovolcanoes, (b) felsic volcanism associated with the large calderas, and (c) total erupted magma volume. 
arc, the magma volume erupted every 100,000-year period (long-term discharge rate of magma) was calculated for each volcano since 2.0 Ma. In the case of South-Hakkoda eruptive episode between 0.65 and $0.40 \mathrm{Ma}$ belonging to Hakkoda volcano (table 1), the erupted volume was estimated to be $52.4 \mathrm{~km}^{3}$ magma from which 10.4, 21.0 and $21.0 \mathrm{~km}^{3}$ magmas can be allocated to the periods of $0.7-0.6,0.6-0.5$ and $0.5-0.4 \mathrm{Ma}$, respectively.

Figure 2 shows plots of cumulative volume associated with large, felsic calderas and andesitic stratovolcanoes as a function of their ages. It indicates that eruptive volume flux of andesitic stratovolcanoes increased significantly after 1.1 Ma. Although flux of eruptive volume from large, felsic calderas increase slightly between 1.4 and 1.0 Ma, the slope, except the slightly anomalous period indicates a constant flux of $\sim 0.1 \mathrm{~km}^{3} / \mathrm{ky}$. Accordingly, volcanism can be divided into two stages: Stage 1 (before ca. 1.0 Ma) is characterized by the predominance of large-volume felsic eruptions forming large calderas, Stage 2 (ca. 1.0 Ma onwards), is dominated by calc-alkaline andesite stratovolcano-building eruption, except for Towada volcano. Stage 2 is marked by a significant increased magmatism in the entire $\mathrm{NE}$ Japan arc. Felsic volcanism, dominant in Stage 1, is believed to have occurred under neutral to weak compressional stress fields associated with gentle uplifting of volcanic arc (Yoshida 2001).
There are two fundamental types of time-volume relationships for volcanic fields: volume-predictable behaviour (linear relationship between the cumulative volume of an eruptive episode plus prior episodes and its timing) and time-predictable behaviour (linear relationship between the timing of an episode and the cumulative volume of prior episodes only). A plot of total volume-age relationship throughout the NE Japan arc suggests that it appears to change somewhat from the time-predictable trend into the volume-predictable trend around 1.0 Ma (figure 2). Valentine and Perry (2007) suggested that eruption marked by the volume-predictable trend occurs in magmatically controlled fields (high magma fluxes), where pressures build up in magma reservoirs, driven primarily by process such as melt accumulation, fractionation, and concentration of volatiles.

Most of the volcanoes exist on uplifted regions forming a topographic high known as the Ou Backbone Range. In addition, $\mathrm{E}-\mathrm{W}$ volcanic zones have been developed with several volcanoes in the rear arc (figure 1). It should be noted that the distribution of volcanoes formed in Stage 1 is limited on the Ou Backbone Range. However, Stage 2 is characterized by volcanism extended to the rear arc, suggesting that Quaternary NE Japan volcanism, stratovolcanoes arranging in E-W direction perpendicular to the volcanic arc, have been established since ca. 1.0 Ma (figure 3).
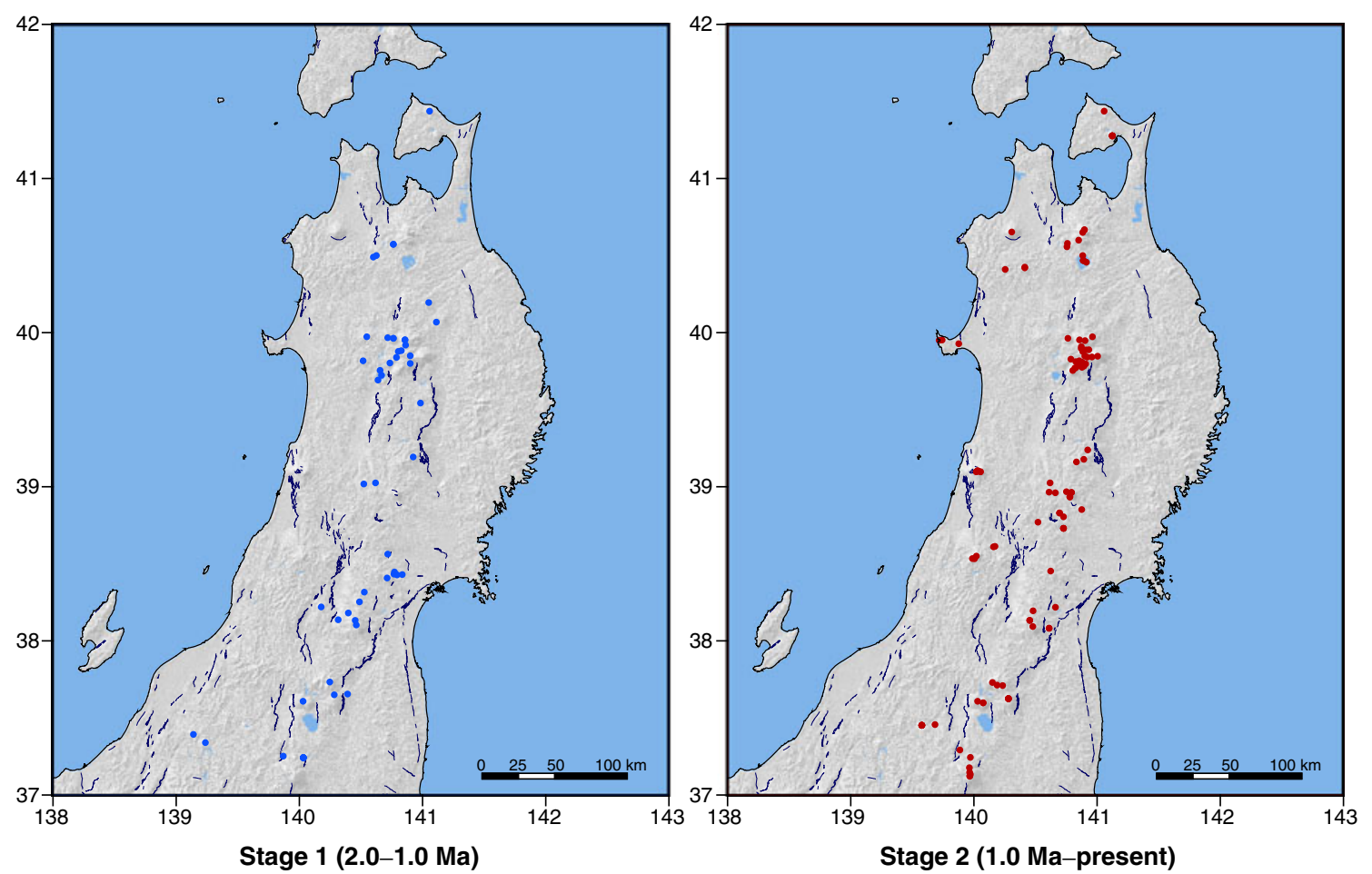

Figure 3. Distribution of eruptive centres for Stage 1 (2.0-1.0 Ma) and Stage 2 (1.0-0 Ma). Also shown in this figure are active faults. 


\section{Discussion and conclusions}

As mentioned in the previous section, the NE Japan arc has been undergoing E-W compression without any significant oblique component during the Quaternary, and reverse faulting has occurred across the entire volcanic arc since about $1.0 \mathrm{Ma}$, resulting in the predominance of the $\mathrm{N}-\mathrm{S}$ trending thrusts with crustal shortening. Contemporaneous changes in volcanism occurred as well; around 1.0 Ma, andesite volcanism with stratovolcanoforming eruptions was initiated. Moreover, magma discharge rate in the entire volcanic arc has rapidly increased since ca. 1.0 Ma. At the same time, the magma underwent a systematic change in chemical composition. A significant volume of medium-K andesite has been erupted along the Ou Backbone Range since 1.0 Ma to 0.7 Ma, together with subordinate low-K andesite (Ban et al. 1992). What are the processes linked to volcanic and tectonic changes around 1.0 Ma in the NE Japan arc? Several researchers insist that the Pacific plate underwent significant changes in the amount and absolute direction of motion between 5 and $2 \mathrm{Ma}$ (Cox and Engebretson 1985; Pollitz 1986). These studies agree on a significant increase in the subduction rate of the Pacific plate, estimated, in the NE Pacific, between $\sim 30$ and $\sim 70 \%$. In consequence, the NE Japan arc has been undergoing active compressional inversion with shortening intensifying over 2.0 Ma.

However, despite compressional stress regime $\left(\sigma_{v}=\sigma_{3}<\sigma_{2}<\sigma_{1}\right)$, a large amount of magma has been extruded since ca. 1.0 Ma. One of the plausible explanations for this contradiction may be the increase in magma generation in the mantle wedge. Numerical simulations considering fluid migration and melting in the mantle wedge above a subducting plate indicate that melt production rates increase with increasing convergence rate (Cagnioncle et al. 2007). In the Cascade volcanic arc, the convergence rate of the Juan de Fuca plate to the North American plate is thought to control the change in eruption rate (Priest 1990). Therefore, despite the overall compressive setting along the NE Japan arc, the increase in volume of magma could be due to the increase in degree of partial melting in the mantle wedge promoted by significantly faster subducting Pacific plate.

In addition, recent experimental modelling provided insight into the upwelling process of magma within brittle crust under a compressive stress regime (Galland et al. 2007). These researchers employed (1) silica powder to replicate a brittle crust and (2) liquid vegetable oil representing magma, to model materials. Under compressional settings using a steadily advancing piston, the silica powder 'crust' shortened as the result of the thrust fault development, which had straight traces at the free surface. When shortening of the silica powder and injection of the oil were simultaneous, magma formed a basal sill and then typically rose along a thrust fault. These results imply that, theoretically, compression favours the emplacement of sills (Hubbert and Willis 1957), but thrust faults then provide vertical tension fractures serving as conduits for magma in the shallow portion of the hanging wall, demonstrating that magma can propagate to the Earth's surface in a tectonic regime characterized by contracting deformation. These structures can be attributed to superposition of a local load, due to the uplifted zone, on the regional stress field (Molnar 1986). Therefore, apparent thrust faulting throughout the $\mathrm{Ou}$ Backbone Range since ca. 1.0 Ma could facilitate the effective transfer of magma from the crust and localize newly formed volcanoes close to thrust faults on $\mathrm{Ou}$ Backbone Range.

Consequently, volcanism associated with andesite stratovolcanoes, and thrust faulting due to tectonic shortening has been clearly active since ca. 1.0 Ma, in the NE Japan arc. This is because the increased melt production rate within the mantle wedge and compressional stress regime is ascribed to a significant increase in the subduction rate of the Pacific plate between 5 and $2 \mathrm{Ma}$. The contractional tectonic settings that form a basal sill for magma prevent magma from reaching the Earth's surface. Nevertheless, some volume of magma from the basal sill could be extruded onto the surface where uplifted mountains, bounded by reverse faults, perpendicular to the volcanic arc, have been established since ca. 1.0 Ma. Rather slow ascent of magma from the basal sill may allow differentiated felsic magma within the upper crust to mix with primary magma arising from the deeper potion, such as lower crust and/or mantle wedge, resulting in the production of calc-alkaline andesite volcanism.

\section{Acknowledgements}

The authors would like to thank T Ohba, M Sasaki and K Akaishi for helpful discussions. Careful reviewing of R I Tilling, S J Day, G F McCrank and the anonymous reviewers has greatly improved the paper.

\section{References}

Acocella V, Yoshida T, Yamada R and Funiciello F 2008 Structural control on Late Miocene to Quaternary volcanism in the NE Honshu arc, Japan; Tectonics 27 TC5008, doi: 10.1029/2008TC002296.

Aoki K and Arai F 2000 Late Quaternary tephrostratigraphy of Marine Core KH94-3, LM-8 off Sanriku, Japan; Quat. Res. 39 107-120. 
Arakawa T, Okajima T, Mizukami K, Shimura S, Miyawaki R, Momose M, Kobayashi M and Yoshida M 2008 Eruption history on Osore-zan Volcano, Shimokita peninsula: Long-term change of magma eruptive rate and eruption style, etc; Abst. Volcanol. Soc. Japan 2008 Fall Meeting A16.

Awata Y and Kakimi T 1985 Quaternary tectonics and damaging earthquakes in northeast Honshu, Japan; Earthq. Predict. Res. 3 231-251.

Ban M, Oba Y, Ishikawa K and Takaoka N 1992 K-Ar dating of Mutsu-Hiuchidake, Osoreyama, Nanashigure, and Aoso volcanoes of the Aoso-Osore volcanic zone - The formation of the present volcanic zonation of the Northeast Japan arc; J. Mineral. Petrol. Econ. Geol. 87 39-49.

Cagnioncle A-M, Parmentier E M and Elkins-Tanton L T 2007 Effect of solid flow above a subducting slab on water distribution and melting at convergent plate boundaries; J. Geophys. Res. 112 B09402, doi: 10.1029/2007JB004934.

Chiba S and Kimura J 2001 Geology and growth history of Bandai volcano, Tohoku-Honshu arc, Japan: Analysis of volcanic activity by tephrochronology; Japan Mag. Mineral. Petrol. Sci. 30 126-156.

Committee for Catalogue of Quaternary Volcanoes in Japan 1999 Catalogue of Quaternary Volcanoes in Japan (Tokyo: Volcanology Society of Japan).

Cox A and Engebretson D 1985 Change in motion of Pacific Plate at 5 Myr BP; Nature 313 472-475.

DeMets C 1992 Oblique convergence and deformation along the Kuril and Japan trenches; J. Geophys. Res. 97 (B12) 17615-17625.

Fujinawa A, Hayashi S and Umeda K 2001a K-Ar ages for lava samples of Adatara volcano: Reexamination of formation history of the volcano; Bull. Volcanol. Soc. Japan 46 95-106.

Fujinawa A, Fujita K, Takahashi M, Umeda K and Hayashi S 2001b Development history of Kurikoma volcano, Northeast Japan; Bull. Volcanol. Soc. Japan 46 269-284.

Galland O, Cobbold P R, de Bremond d'Ars J and Hallot E 2007 Rise and emplacement of magma during horizontal shortening of the brittle crust: Insights from experimental modeling; J. Geophys. Res. 112 B06402, doi: 10.1029/ 2006JB004604.

Hamilton W B 1995 Subduction systems and magmatism; In: Volcanism Associated with Extension at Consuming Plate Margins (ed.) Smellie J L, Geol. Soc. London, Spec. Publ. 81 3-28.

Hasegawa A, Umino N and Takagi A 1978 Double-planed structure of the deep seismic zone in the northeastern Japan arc; Tectonophys. 47 43-58.

Hasegawa A, Nakajima J, Umino N and Miura S 2005 Deep structure of the northeastern Japan arc and its implications for crustal deformation and shallow seismic activity; Tectonophys. 403 59-75.

Hubbert M K and Willis D G 1957 Mechanics of hydraulic fracturing; In: Structural geology (ed.) Hubbert M K (New York: Hafner Publishing Company), pp. 175-190.

Kano K, Ohguchi T, Hayashi S and Yanai K 2007 Tazawako caldera and its eruption products; Abst. Geol. Soc. Japan 114th Annual Meeting O-17.

Jolivet L, Tamaki K and Fournier M 1994 Japan Sea, opening history and mechanism: A synthesis; J. Geophys. Res. 99(B11) 22,237-22,259.

Kimura J and Yoshida T 2006 Contribution of slab fluid, mantle wedge and crust to the origin of Quaternary lavas in the NE Japan Arc; J. Petrol. 47 2185-2232.

Mimura K 2001 K-Ar dating of Nanatsumori volcanic rock, Kamurodake and Aoso volcanoes along the Quaternary volcanic front of northeast Japan; Bull. Volcanol. Soc. Japan 52 309-313.
Mimura K 2002 Geology and radiometric ages of Nekoma Volcano, Northeast Japan; Bull. Volcanol. Soc. Japan 47 $217-225$.

Mimura K and Kano K 2000 Stratigraphy and history of Shirataka Volcano, NE Japan; Bull. Volcanol. Soc. Japan 45 13-23.

Molnar P 1986 The structure of mountain ranges; Sci. Amer. 255 64-73.

Nakamura K 1964 Volcano-stratigraphic study of Oshima volcano, Izu; Bull. Earthq. Res. Inst. 42 649-728.

Nakata T and Imaizumi T 2002 Digital active fault map of Japan (DVD-ROM) (Tokyo: University of Tokyo Press), p. 60 .

NEDO 1990 Explanatory text of the volcano-geological map and compiled geological map of Nasu geothermal area (Tokyo: New Energy and Industrial Technology Development Organization), 68p.

Nozawa A 2001 Volcanic sequence of caldera-fill deposits and structure of Okiura caldera, Hakkoda geothermal area, Northeast Japan; J. Geol. Soc. Japan 107 413-431.

Ohba T, Hayashi S and Umeda K 2003 K-Ar ages of volcanic rocks from northern vicinity of Matsukawa geothermal field, Iwate Prefecture; Bull. Volcanol. Soc. Japan 48 $367-374$

Ohguchi T, Yoshida T and Okami K 1989 Historical change of the Neogene and Quaternary volcanic field in the Northeast Honshu arc, Japan; Mem. Geol. Soc. Japan 32 431-455.

Ono K, Soya T and Mimura K 1981 Volcanoes of Japan (2nd edn). 1: 2,000,000 map series, no. 11 (Tsukuba: Geological Survey of Japan).

Otsuki K 1990 Neogene tectonic stress fields of northeast Honshu arc and implications for plate boundary conditions; Tectonophys. 181 151-164.

Otsuki K, Nakata T and Imaizumi T 1977 Quaternary crustal movements and block model in the southeastern region of the Northeast Japan; Earth Sci. 31 1-14.

Pollitz F F 1986 Pliocene change in Pacific Plate motion; Nature 320 738-741.

Priest G 1990 Volcanic and tectonic evolution of the cascade volcanic arc, Central Oregon; J. Geophys. Res. 95 (B12) $19,583-19,599$.

Sagiya T, Miyazaki S and Tada T 2000 Continuous GPS arrays and present-day crustal deformation of Japan; Pure Appl. Geophys. 157 2303-2322.

Sakuyama M and Nesbitt R W 1986 Geochemistry of the Quaternary volcanic rocks of the Northeast Japan arc; J. Volcanol. Geotherm. Res. 29 413-450.

Sato H 1994 The relationship between late Cenozoic tectonic events and stress field and basin development in northeast Japan; J. Geophys. Res. 99(B11) 22261-22274.

Smith R L 1979 Ash-flow magmatism; Geol. Soc. Am. Bull., Spec. Paper 180 5-27.

Suzuki T, Eden D, Danhara T and Fujiwara O 2005 Correlation of the Hakkoda-Kokumoto Tephra, a widespread Middle Pleistocene tephra erupted from the Hakkoda Caldera, northeast Japan; The Island Arc 14 666-678.

Taira A 2001 Tectonic evolution of the Japanese island arc system; Annu. Rev. Earth Planet. Sci. 29 109-134.

Tamura Y, Tatsumi Y, Zhao D, Kido Y and Shukuno H 2002 Hot fingers in the mantle wedge: New insights into magma genesis in subduction zones; Earth Planet. Sci. Lett. 197 105-116.

Tsuchiya N, Itoh J I, Seki Y and Iwaya T 1997 Geology of the Iwagasaki District. Quadrangle Series, Scale 1:50,000 (Tsukuba: Geological Survey of Japan).

Umeda K and Danhara T 2008 Zircon fission-track ages for pyroclastics from the Mutsu-Hiuchidake volcano and the basement rocks, Northeast Japan: Re-examination of the 
latest volcanic history; Japan Mag. Mineral. Petrol. Sci. 37 131-136.

Umeda K, Hayashi S, Ban M, Sasaki M, Oba T and Akaishi K 1999 Sequence of volcanism and tectonics during the last 2.0 million years along the volcanic front in Tohoku district, NE Japan; Bull. Volcanol. Soc. Japan 44 233-249.

Valentine G A and Perry F V 2007 Tectonically controlled, time-predictable basaltic volcanism from a lithospheric mantle source (central Basin and Range Province, USA); Earth Planet. Sci. Lett. 261 201-216.

Walker G P L 1980 The Taupo pumice: Product of the most powerful known (ultraplinian) eruption?; J. Volcanol. Geotherm. Res. 8 69-94.

Watanabe T, Koyaguchi T and Seno T 1999 Tectonic stress controls on ascent and emplacement of magmas; J. Volcanol. Geotherm. Res. 91 65-78.

Yamamoto T 2003 Eruptive history of Numazawa volcano, NE Japan: New study of the stratigraphy, eruption ages, and eruption volumes of the products; Bull. Geol. Surv. Japan 54 323-340.

Yamamoto $\mathrm{T}$ and Komazawa M 2004 Geology of the Miyashita District. Quadrangle Series, 1:50,000 (Tsukuba: Geological Survey of Japan).

Yashima R $1990 \mathrm{~K}-\mathrm{Ar}$ Ages of Pliocene volcanic rocks in Northeast Honshu Arc, Japan: Ajarayama Andesite, Aonokimori Andesite, Nanatsumori Dacite and Sasamoriyama Andesite; Chikyu Kagaku 44 150-153.

Yoshida T 2001 The evolution of arc magmatism in the NE Honshu arc, Japan; Tohoku Geophys. J. 36 131-149.

Yoshida H and Takahashi M 1991 Geology of the eastern part of the Shirakawa pyroclastic flow field; J. Geol. Soc. Japan 97 231-249.

Zhao D, Hasegawa A and Horiuchi S 1992 Tomographic imaging of $\mathrm{P}$ and $\mathrm{S}$ wave velocity structure beneath Northeastern Japan; J. Geophys. Res. 97 (B13) 19,90919,928 . 